\title{
17. PETROLOGY AND MAGMATIC AFFINITY OF THE NORTH D'ENTRECASTEAUX RIDGE, CENTRAL NEW HEBRIDES TRENCH, SITE $828^{1}$
}

\author{
M. Coltorti, ${ }^{2}$ T. Hasenaka, ${ }^{3}$ L. Briqueu, ${ }^{4}$ P.E. Baker, ${ }^{5}$ and F. Siena ${ }^{2}$
}

\begin{abstract}
Petrography, major and trace elements, mineral chemistry, and $\mathrm{Sr}, \mathrm{Nd}$, and $\mathrm{Pb}$ isotopic ratios are reported for igneous rocks drilled on the northern flank of the North d'Entrecasteaux Ridge (NDR) during Ocean Drilling Program (ODP) Leg 134 Site 828. These rocks comprise a breccia unit beneath a middle Eocene foraminiferal ooze. Both geophysical characteristics and the variety of volcanic rocks found at the bottom of Holes $828 \mathrm{~A}$ and $828 \mathrm{~B}$ indicate that a very immature breccia or scree deposit was sampled.

Basalts are moderately to highly altered, but primary textures are well preserved. Two groups with different magmatic affinities, unrelated to the stratigraphic height, have been distinguished. One group consists of aphyric to sparsely plagioclase + clinopyroxene-phyric basalts, characterized by high $\mathrm{TiO}_{2}(\sim 2 \mathrm{wt} \%)$ and low $\mathrm{Al}_{2} \mathrm{O}_{3}$ (less than $\left.15 \mathrm{wt} \%\right)$ contents, with flat MORB-normalized incompatible element patterns and LREE-depleted chondrite-normalized REE patterns. This group resembles N-MORB.

The other group comprises moderately to highly olivine + plagioclase-phyric basalts with low $\mathrm{TiO}_{2}(<1 \mathrm{wt} \%)$ and high $\mathrm{Al}_{2} \mathrm{O}_{3}$ (usually $>15 \mathrm{wt} \%$ ) contents, and marked HFSE depletion and LFSE enrichment. Some lavas in this group are picritic, with relatively high modal olivine abundances, and $\mathrm{MgO}$ contents up to $15 \mathrm{wt} \%$. Both the basalts and picritic basalts of this group reflect an influence by subduction-related processes, and have compositions transitional between MORB and IAT. Lavas with similar geochemical features have been reported from small back-arc basins such as the Mariana Trough, Lau Basin, Sulu Sea, and the North Fiji Basin and are referred to as back-arc basin basalts. However, regional tectonic considerations suggest that the spreading that produced these backarc basin basalts may have occurred in the forearc region of the southwest-facing island arc that existed in this region in the Eocene.
\end{abstract}

\section{INTRODUCTION}

One of the major objectives of Ocean Drilling Program (ODP) Leg 134 was to investigate the nature and tectonic evolution of the d'Entrecasteaux Zone (DEZ; previously referred to as d'Entrecasteaux Fracture Zone; Kroenke, 1984), a zone of aseismic ridges that is colliding with the Western Belt islands (Espiritu Santo and Malakula) of the New Hebrides Island Arc.

The DEZ comprises an east-trending ridge (North d'Entrecasteaux Ridge, NDR) that is elevated 2 to $4 \mathrm{~km}$ above the seafloor, and a seamount chain (South d'Entrecasteaux Chain, SDC), which includes Sabine Bank and Bougainville Guyot (see also Baker et al., this volume). It exceeds $100 \mathrm{~km}$ in width and extends more than $600 \mathrm{~km}$ from New Caledonia to Vanuatu (Fig. 1).

Before ODP Leg 134, samples from the DEZ had been obtained by dredging (Maillet et al., 1983) and, more recently, by submersible (Collot et al., 1989, 1992). Drilling at Site 286 in the North Loyalty Basin, located approximately $75 \mathrm{~km}$ south of Sabine Bank, was carried out during Deep Sea Drilling Project (DSDP) Leg 30 (Fig. 1).

Petrographic and geochemical data for dredged rocks indicated a mid-ocean ridge basalt (MORB) affinity. Fission track age determinations range between 36 and $56 \mathrm{Ma}$ (i.e., between the Eocene/Oligocene and Paleocene/Eocene boundaries; Maillet et al., 1983). In contrast, a clear calc-alkaline affinity was recognized for igneous clasts in the middle to upper Eocene andesitic conglomerate of Subunit 3B at Site 286 (Stoeser, 1975). Because Site 286 bottomed in ocean-floor gabbroic rocks, the provenance of these andesitic clasts may lie in the

\footnotetext{
${ }^{1}$ Greene, H.G., Collot, J.-Y., Stokking, L.B., et al., 1994. Proc. ODP, Sci. Results, 134: College Station, TX (Ocean Drilling Program). Italy.

${ }^{2}$ Mineralogy Institute, Ferrara University, C.so Ercole $1^{\circ}$ d'Este, 32, 44100 Ferrara,

${ }^{3}$ Institute of Mineralogy, Petrology and Economic Geology, Faculty of Science, Tohoku University, Aoba, Sendai, Miyagi 980, Japan.

${ }^{4}$ CNRS France, Laboratoire de Géochimie Isotopique, U.S.T.L., C.P. 066, 34095 Montpellier Cedex 2, France.

${ }^{5}$ Department of Earth Sciences, University of Leeds, Leeds LS2 9JT, United Kingdom.
}

southern portion of DEZ. Data from Maillet et al. (1983) and Burne et al. (1988) suggests that the SDC represents the remnant of an Eocene proto-island arc. Baker et al. (this volume) document andesites with island arc tholeiitic (IAT) affinity at Site 831, and Collot et al. (1992) recovered igneous rocks from submersible surveys confirming an arc geochemical signature for Bougainville Guyot. In this framework, the NDR could be interpreted either as a slow-spreading ridge close to an active island arc (Maillet et al., 1983) or as an intraoceanic arc produced during south-dipping pre-Miocene subduction (Collot et al., 1985, 1992; Burne et al., 1988).

Petrography, major and trace element (including REE) analyses, and $\mathrm{Sr}, \mathrm{Nd}$, and $\mathrm{Pb}$ isotopic determinations are presented in this paper to elucidate the nature and magmatic affinity of the igneous rocks recovered from Site 828 . This study also contributes to the interpretation of the volcanic rocks (particularly those at Site 829; Coltorti et al., this volume) drilled in the Vanuatu forearc region, and the process of collision/accretion between the NDR (Australia-India Plate) and the presently active volcanic chain of the New Hebrides Island Arc; and the tectono-magmatic significance of the DEZ, including the geochemical affinity and volcanic evolution of its southern part, particularly Bougainville Guyot (Site 831; Baker et al., this volume).

\section{SITE LOCATION AND STRATIGRAPHY}

During Leg 134 two sites were drilled in the DEZ: Site 828 on the northern flank of the NDR, and Site 831 on the summit of Bougainville Guyot. Site 828 is located about $2 \mathrm{~km}$ west of the trace of the New Hebrides trench and about $40 \mathrm{~km}$ from the western coast of Espiritu Santo island (Fig. 1).

Two holes were drilled at Site 828 : Hole $828 \mathrm{~A}\left(15^{\circ} 17.34^{\prime} \mathrm{S}, 166^{\circ}\right.$ $\left.17.04^{\prime} \mathrm{E}\right)$ and Hole $828 \mathrm{~B}\left(15^{\circ} 17.26^{\prime} \mathrm{S}, 166^{\circ} 16.96^{\prime} \mathrm{E}\right)$. They are situated on a flat, terrace-like surface at $\sim 3080 \mathrm{mbsl}$ ( $3087 \mathrm{~m}$ for Hole $828 \mathrm{~A} ; 3082 \mathrm{~m}$ for Hole $828 \mathrm{~B}$ ), about $1000 \mathrm{~m}$ below the highest part of the NDR. Geophysical surveys identified a basin-like feature just below the sedimentary cover and above the presumed basement approximately 300 mbsf. Hole $828 \mathrm{~A}$ cored $111.4 \mathrm{~m}$, recovering 101.34 


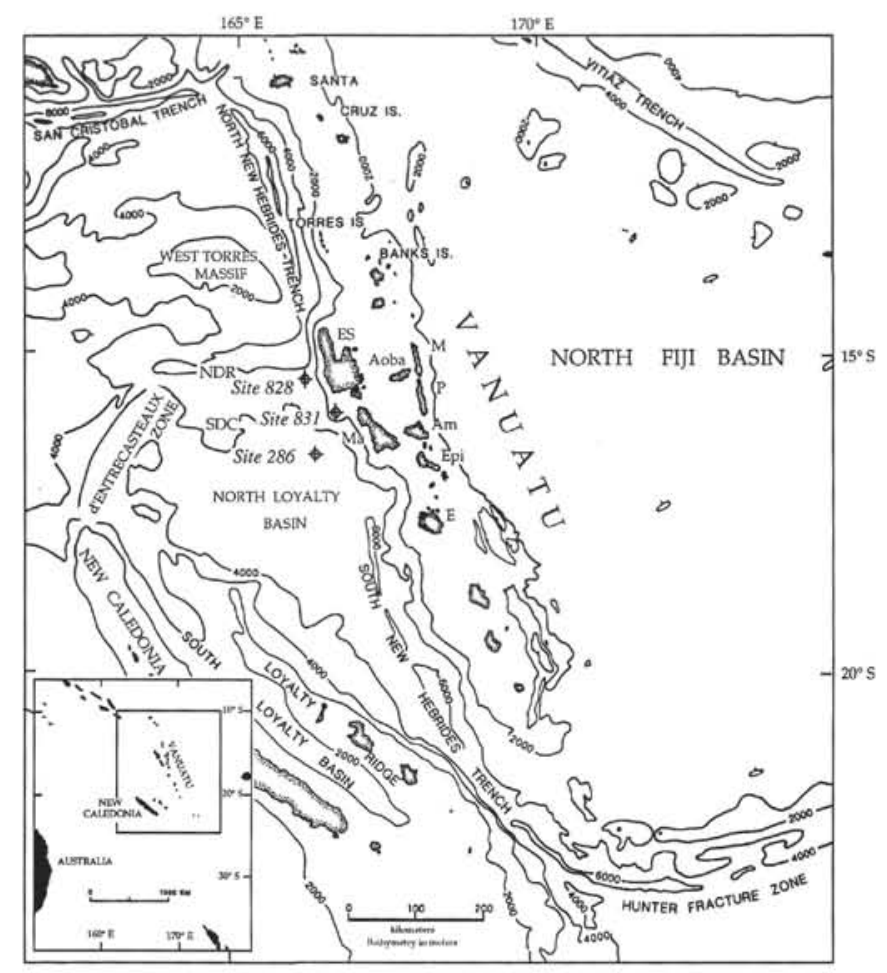

Figure 1. Geographic features of the New Hebrides Island Arc and location of ODP Sites 828 and 831 , and DSDP Site 286. NDR = North d'Entrecasteaux Ridge $;$ SDC $=$ South d'Entrecasteaux Chain $;$ ES = Espiritu Santo; $\mathrm{Ma}=$ Malakula; $\mathrm{M}=$ Maewo; $\mathrm{P}=$ Pentecost $\mathrm{Am}=$ Ambrym $; \mathrm{E}=$ Efate.

$\mathrm{m}$, while Hole $828 \mathrm{~B}$ penetrated only $129.0 \mathrm{~m}$, coring $39.0 \mathrm{~m}$ to recover $7.92 \mathrm{~m}$.

Four lithostratigraphic units primarily from Hole $829 \mathrm{~A}$ have been described. Unit I ( $0-58.7 \mathrm{mbs})$ is composed of a Pleistocene sequence of volcanic silt, with interbeds of graded volcaniclastic sand to silt with foraminifers. Unit II (58.7-69.3 mbsf) consists of totally unconsolidated foraminiferal ooze with late Miocene or early Pliocene to Pleistocene nannofossils. Unit III (69.3-90.8 mbsf) is a highly bioturbated, early to late Oligocene foraminiferal ooze (Reid et al., this volume). The Unit II/III contact marks a biostratigraphic hiatus, and most of the Miocene is missing across this boundary. Unit IV (90.8-111.4 mbsf) consists of volcanic breccia and brecciated lava flow deposits. Igneous clasts, ranging in size from 2 to $5 \mathrm{~cm}$, are angular to subrounded and embedded in a silty to clayey sand matrix. A few middle Eocene planktonic foraminifera were found in the core catcher. Igneous rocks were recovered at the bottom of both holes: $7.4 \mathrm{~m}$ in Hole $828 \mathrm{~A}$ and $0.16 \mathrm{~m}$ in Hole $828 \mathrm{~B}$. The wide variety of volcanic rocks encountered indicates that the "basement," for which more homogeneous basalts might be anticipated, was not penetrated. The rocks sampled probably represent a very immature breccia or scree deposit.

\section{ANALYTICAL METHODS}

$\mathrm{X}$-ray fluorescence (XRF), major and trace element analyses (excluding rare earth elements, or REE) were conducted aboard the JOIDES Resolution, at the Institute of Mineralogy, Ferrara University (Italy), and at the Institute of Mineralogy, Petrology and Economic Geology, Tohoku University (Japan). Shipboard analyses were performed on glass discs for major elements and on powder pellets for trace elements, using a Compton scattering technique for matrix absorbing corrections (Reynolds, 1967). Major element data are con- sidered accurate between $1 \%$ and $5 \%$, whereas trace element accuracy varies from $2 \%$ to $10 \%$, except for $\mathrm{Ba}$ and $\mathrm{Ce}$, which exceed $10 \%$ (see "Explanatory Notes," Collot, Greene, Stokking, et al., 1992). Analyses from Ferrara University were performed on powder pellets using a wavelength-dispersive automated Philips PW 1400 spectrometer. Major elements were determined by a full matrix correction procedure (Franzini et al., 1975), whereas for trace elements experimentally determined correction coefficients were used (Leoni and Saitta, 1976). Accuracy and precision for major elements are estimated better than $3 \%$ for $\mathrm{Si}, \mathrm{Ti}, \mathrm{Fe}, \mathrm{Ca}$, and $\mathrm{K}$, and $7 \%$ for $\mathrm{Mg}, \mathrm{Na}$, $\mathrm{Al}, \mathrm{Mn}$, and $\mathrm{P}$; for trace elements (above $10 \mathrm{ppm}$ ), they are better than $7 \%$ for $\mathrm{Rb}, \mathrm{Nb}, \mathrm{Y}, \mathrm{Sr}, \mathrm{V}$, and $15 \%$ for $\mathrm{Zr}, \mathrm{La}, \mathrm{Ce}, \mathrm{Ba}, \mathrm{Ni}, \mathrm{Co}$, and $\mathrm{Cr}$. Analyses of reference standards AGV 1 and $\mathrm{BR}$ are reported in Table 1 for comparison. Loss on ignition (LOI) was determined by a gravimetric method. REE and Y were determined at the Centre de Recherches Pétrographiques et Géochimiques of Nancy (France) by inductively coupled plasma (ICP) emission spectrometry with an accuracy of $15 \%$ for $\mathrm{Yb}$ and $\mathrm{Lu}$ and better than $8 \%$ for the other REE (see analyses of reference standards from Roelandts and Michel, 1986). Analytical methods for analyses performed at Tohoku University (including REE) are described in Hasenaka et al. (this volume).

In order to facilitate comparison between analyses from different laboratories, $\mathrm{Fe}_{2} \mathrm{O}_{3}$ was calculated as $0.15 \mathrm{FeO}$, and analyses were recalculated to $100 \%$ on an anhydrous basis.

Analyses carried out in the different laboratories display very reasonable agreement. Some discrepancies can be observed in the $\mathrm{SiO}_{2}$ and $\mathrm{CaO}$ contents, and are probably related to variable degrees of alteration, even in samples very close together in the core (most of them as clasts). Trace elements, particularly those considered unaffected by secondary remobilization, show good agreement, except $\mathrm{Y}$ by ICP, which is systematically lower (about 15\%) than by XRF. Isotopic analyses were carried out at the Laboratoire de Géochimique Isotopique, Université de Montpellier, after leaching with $2 \mathrm{~N} \mathrm{HF}+$ $0.5 \mathrm{~N} \mathrm{HBr}$ mixture and cold $2.5 \mathrm{~N} \mathrm{HCl}$ (see the analytical methods section in Briqueu et al., this volume).

Minerals were analyzed at the University of Leeds (UK) using a CAMECA SX-50 electron-probe microanalyzer fitted with three wavelength-dispersive spectrometers and a LINK 10/55S energy-dispersive system, at an accelerating voltage of $15 \mathrm{kV}$, and specimen current of 15 $\mathrm{nA}$. Natural silicates and oxides standards were used, and the raw data were corrected using CAMECA proprietary software. For more details see the analytical methods section in Baker et al. (this volume).

\section{PETROGRAPHY}

All the igneous rocks recovered, except one (Sample 134-828B-2RCC, $3-6 \mathrm{~cm}$ ), are basalts. They are predominantly non- (or sparsely) vesicular, generally hypocrystalline, fine to medium grained, and aphyric or moderately to highly porphyritic. Groundmasses have intersertal to intergranular textures, and hyalopilitic textures are common where glass is particularly abundant. Microlites of plagioclase often occur as radiating or feathery aggregates. Textural relationships indicate that the crystallization sequence is olivine then plagioclase, followed by clinopyroxene, and finally Fe-Ti oxides. Primary textures are well preserved, despite the high degree of alteration. Extensive oxidation has resulted in reddish/black halos and patchy development of $\mathrm{Fe}$-hydroxides. Alteration products include clay minerals as the devitrification products of glassy mesostasis, serpentine after olivine, chlorite, minor epidote, and calcite after mafic phases and, more rarely, also after plagioclase. Crosscutting veins and fractures of various widths $(0.3-2.0 \mathrm{~mm})$ are infilled with calcite, smectites, and minor zeolites. These secondary assemblages are typical of low-temperature submarine alteration. The occurrence of zeolites and the local development of chlorite and epidote may be attributed from prehnite-pumpellyite to lower greenschist metamorphic facies (Elthon, 1981; Alt et al., 1986). 
Table 1. Major trace element and $\mathrm{Sr}, \mathrm{Nd}, \mathrm{Pb}$ isotopic analyses of igneous rocks recovered from Site 828.

\begin{tabular}{|c|c|c|c|c|c|c|c|c|c|c|c|c|c|c|c|c|c|c|c|c|}
\hline $\begin{array}{l}\text { Hole: } \\
\text { Core, section: } \\
\text { Interval (cm): } \\
\text { Depth (mbsf): } \\
\text { Rock type: } \\
\text { Analysis location: }\end{array}$ & $\begin{array}{l}828 \mathrm{~A} \\
12 \mathrm{X}-1 \\
22-24 \\
95.62 \\
\text { Basalt } \\
\text { F }\end{array}$ & $\begin{array}{l}828 \mathrm{~A} \\
12 \mathrm{X}-1 \\
24-26 \\
95.64 \\
\text { Basalt } \\
\mathrm{J}\end{array}$ & $\begin{array}{l}828 \mathrm{~A} \\
12 \mathrm{X}-1 \\
29-31 \\
95.69 \\
\text { Basalt } \\
\text { F }\end{array}$ & $\begin{array}{c}828 \mathrm{~A} \\
12 \mathrm{X}-1 \\
31-35 \\
95.71 \\
\text { Basalt } \\
\mathrm{T}\end{array}$ & $\begin{array}{c}828 \mathrm{~A} \\
13 \mathrm{X}-1 \\
0-5 \\
97.40 \\
\text { Basalt } \\
\mathrm{F}\end{array}$ & $\begin{array}{c}828 \mathrm{~B} \\
2 \mathrm{R}-\mathrm{CC} \\
3-6 \\
100.03 \\
\text { Rhyolite } \\
\text { F }\end{array}$ & $\begin{array}{c}828 \mathrm{~A} \\
15 \mathrm{~N}-1 \\
20-22 \\
108.00 \\
\text { e Basalt } \\
\text { F }\end{array}$ & $\begin{array}{c}828 \mathrm{~A} \\
15 \mathrm{~N}-1 \\
23-25 \\
108.03 \\
\text { Basalt } \\
\text { J }\end{array}$ & $\begin{array}{c}828 \mathrm{~A} \\
15 \mathrm{~N}-1 \\
48-52 \\
108.28 \\
\text { Basalt } \\
\quad \mathrm{F}\end{array}$ & $\begin{array}{l}828 \mathrm{~A} \\
15 \mathrm{~N}-1 \\
52-55 \\
108.32 \\
\text { Basalt } \\
\quad \mathrm{T}\end{array}$ & $\begin{array}{c}828 \mathrm{~A} \\
15 \mathrm{~N}-1 \\
119-120 \\
108.99 \\
\text { Basalt } \\
\text { F }\end{array}$ & $\begin{array}{c}828 \mathrm{~A} \\
15 \mathrm{~N}-1 \\
122-123 \\
109.02 \\
\text { Basalt } \\
\text { J }\end{array}$ & $\begin{array}{c}828 \mathrm{~A} \\
15 \mathrm{~N}-2 \\
3-4 \\
309.33 \\
\text { Basalt } \\
\mathrm{J}\end{array}$ & $\begin{array}{c}828 \mathrm{~A} \\
15 \mathrm{~N}-2 \\
7-9 \\
109.37 \\
\text { Basalt } \\
\text { F }\end{array}$ & $\begin{array}{c}828 \mathrm{~B} \\
3 \mathrm{R}-\mathrm{CC} \\
6-7 \\
109.76 \\
\text { Dolerite } \\
\text { F }\end{array}$ & $\begin{array}{c}828 \mathrm{~A} \\
15 \mathrm{~N}-2 \\
62-63 \\
109.92 \\
\text { Basalt } \\
\mathrm{J}\end{array}$ & $\begin{array}{c}828 \mathrm{~A} \\
15 \mathrm{~N}-2 \\
63-67 \\
109.93 \\
\text { Basalt } \\
\text { T }\end{array}$ & $\begin{array}{c}828 \mathrm{~A} \\
15 \mathrm{~N}-2 \\
70-73 \\
110.00 \\
\text { Basalt } \\
\text { T }\end{array}$ & $\begin{array}{l}\text { AGV } 1 \\
\text { Andesite } \\
\text { F }\end{array}$ & $\begin{array}{c}\text { Basalt } \\
\mathrm{F}\end{array}$ \\
\hline $\begin{array}{l}\mathrm{SiO}_{2} \\
\mathrm{TiO}_{2} \\
\mathrm{Al}_{2} \mathrm{O}_{3} \\
\mathrm{Fe}_{2} \mathrm{O}_{3} \\
\mathrm{FeO} \\
\mathrm{MnO} \\
\mathrm{MgO} \\
\mathrm{CaO} \\
\mathrm{Na}_{2} \mathrm{O} \\
\mathrm{K}_{2} \mathrm{O} \\
\mathrm{P}_{2} \mathrm{O}_{5} \\
\mathrm{LOI}\end{array}$ & $\begin{array}{r}45.04 \\
0.77 \\
14.91 \\
1.16 \\
6.96 \\
0.15 \\
10.67 \\
16.59 \\
3.50 \\
0.12 \\
0.06 \\
9.60\end{array}$ & $\begin{array}{r}50.87 \\
0.73 \\
16.51 \\
1.16 \\
6.99 \\
0.15 \\
9.06 \\
10.22 \\
4.17 \\
0.10 \\
0.04 \\
6.77\end{array}$ & $\begin{array}{r}48.78 \\
0.83 \\
15.12 \\
1.22 \\
7.34 \\
0.16 \\
11.58 \\
10.82 \\
3.94 \\
0.15 \\
0.06 \\
5.88\end{array}$ & $\begin{array}{r}45.38 \\
0.64 \\
16.25 \\
1.10 \\
6.62 \\
0.13 \\
6.44 \\
19.82 \\
3.48 \\
0.10 \\
0.05 \\
-\end{array}$ & $\begin{array}{r}49.45 \\
0.79 \\
15.33 \\
1.13 \\
6.79 \\
0.15 \\
10.00 \\
11.85 \\
4.33 \\
0.13 \\
0.06 \\
6.01\end{array}$ & $\begin{array}{r}76.97 \\
0.21 \\
11.13 \\
0.33 \\
2.00 \\
0.06 \\
2.50 \\
0.48 \\
6.24 \\
0.05 \\
0.03 \\
1.23\end{array}$ & $\begin{array}{r}48.06 \\
0.83 \\
17.39 \\
1.11 \\
6.66 \\
0.14 \\
14.92 \\
7.34 \\
3.10 \\
0.40 \\
0.05 \\
4.78\end{array}$ & $\begin{array}{r}49.51 \\
0.81 \\
18.16 \\
1.14 \\
6.85 \\
0.13 \\
12.28 \\
7.05 \\
3.50 \\
0.51 \\
0.05 \\
6.19\end{array}$ & $\begin{array}{r}46.61 \\
0.80 \\
16.31 \\
1.10 \\
6.58 \\
0.15 \\
14.76 \\
9.11 \\
3.91 \\
0.59 \\
0.08 \\
8.25\end{array}$ & $\begin{array}{r}49.34 \\
0.78 \\
17.53 \\
1.22 \\
7.34 \\
0.14 \\
11.24 \\
6.28 \\
5.03 \\
1.05 \\
0.05 \\
-\end{array}$ & $\begin{array}{r}51.84 \\
1.93 \\
14.03 \\
1.70 \\
10.20 \\
0.17 \\
8.10 \\
5.68 \\
5.81 \\
0.35 \\
0.18 \\
1.95\end{array}$ & $\begin{array}{r}53.45 \\
1.92 \\
14.41 \\
1.68 \\
10.07 \\
0.19 \\
6.68 \\
5.45 \\
5.74 \\
0.24 \\
0.17 \\
3.41\end{array}$ & $\begin{array}{r}52.66 \\
1.90 \\
14.61 \\
1.75 \\
10.48 \\
0.20 \\
6.73 \\
5.59 \\
5.56 \\
0.38 \\
0.15 \\
3.70\end{array}$ & $\begin{array}{r}50.51 \\
2.00 \\
14.28 \\
1.85 \\
11.10 \\
0.18 \\
8.45 \\
5.61 \\
5.44 \\
0.40 \\
0.16 \\
2.60\end{array}$ & $\begin{array}{r}50.72 \\
1.05 \\
16.32 \\
1.30 \\
7.82 \\
0.19 \\
9.43 \\
8.11 \\
4.66 \\
0.31 \\
0.08 \\
3.10\end{array}$ & $\begin{array}{r}48.72 \\
0.82 \\
17.87 \\
1.04 \\
6.23 \\
0.15 \\
12.95 \\
8.32 \\
2.99 \\
0.87 \\
0.04 \\
6.77\end{array}$ & $\begin{array}{r}48.92 \\
0.81 \\
17.75 \\
1.22 \\
7.33 \\
0.15 \\
11.83 \\
6.36 \\
4.79 \\
0.80 \\
0.05 \\
-\end{array}$ & $\begin{array}{r}49.09 \\
0.82 \\
17.56 \\
1.20 \\
7.18 \\
0.17 \\
11.08 \\
7.68 \\
4.69 \\
0.49 \\
0.05 \\
-\end{array}$ & $\begin{array}{l}0.10 \\
1.18 \\
4.87 \\
4.26 \\
2.83 \\
0.48 \\
-\end{array}$ & $\begin{array}{r}0.17 \\
13.57 \\
13.30 \\
3.15 \\
1.39 \\
1.31 \\
-\end{array}$ \\
\hline $\mathrm{mgv}$ & 0.73 & 0.70 & 0.74 & 0.63 & 0.72 & 0.69 & 0.80 & 0.76 & 0.80 & 0.73 & 0.59 & 0.54 & 0.53 & 0.58 & 0.68 & 0.79 & 0.74 & 0.73 & & \\
\hline $\begin{array}{l}\mathrm{Ni} \\
\mathrm{Co} \\
\mathrm{Cr} \\
\mathrm{V} \\
\mathrm{Rb} \\
\mathrm{Sr} \\
\mathrm{Ba} \\
\mathrm{Zr} \\
\mathrm{Nb} \\
\mathrm{La} \\
\mathrm{Ce} \\
\mathrm{Y}\end{array}$ & $\begin{array}{r}135 \\
38 \\
362 \\
200 \\
1 \\
89 \\
17 \\
24 \\
2 \\
\text { n.d. } \\
5 \\
26\end{array}$ & $\begin{array}{c}131 \\
344 \\
204 \\
2 \\
76 \\
24- \\
1 \\
1- \\
6 \\
18\end{array}$ & $\begin{array}{r}143 \\
44 \\
404 \\
224 \\
2 \\
74 \\
29 \\
28 \\
2 \\
\text { n.d. } \\
6 \\
22\end{array}$ & $\begin{array}{r}98 \\
28 \\
290 \\
188 \\
1 \\
68 \\
7 \\
26 \\
1 \\
- \\
18\end{array}$ & $\begin{array}{r}157 \\
43 \\
394 \\
238 \\
2 \\
25 \\
65 \\
23 \\
21 \\
1 \\
1 \\
4 \\
20\end{array}$ & $\begin{array}{c}5 \\
7 \\
11 \\
33 \\
\text { n.d. } \\
41 \\
18 \\
78 \\
2 \\
5 \\
6 \\
37\end{array}$ & $\begin{array}{r}169 \\
44 \\
506 \\
210 \\
2 \\
119 \\
48 \\
41 \\
1 \\
1 \\
4 \\
40\end{array}$ & $\begin{array}{r}143 \\
405 \\
214 \\
3 \\
119 \\
18 \\
41 \\
1 \\
4 \\
4 \\
17\end{array}$ & $\begin{array}{r}194 \\
43 \\
456 \\
178 \\
7 \\
113 \\
32 \\
40 \\
2 \\
2 \\
7 \\
18\end{array}$ & $\begin{array}{r}172 \\
52 \\
514 \\
195 \\
14 \\
121 \\
42 \\
43 \\
1 \\
= \\
18\end{array}$ & $\begin{array}{r}104 \\
43 \\
200 \\
362 \\
5 \\
91 \\
30 \\
173 \\
3 \\
6 \\
15 \\
46\end{array}$ & $\begin{array}{r}76 \\
118 \\
319 \\
2 \\
94 \\
8 \\
124 \\
3 \\
10 \\
10 \\
40\end{array}$ & $\begin{array}{r}94 \\
123 \\
317 \\
4 \\
94 \\
7 \\
117 \\
3 \\
18 \\
18 \\
40\end{array}$ & $\begin{array}{r}112 \\
46 \\
222 \\
366 \\
4 \\
100 \\
32 \\
161 \\
5 \\
7 \\
14 \\
47\end{array}$ & $\begin{array}{c}63 \\
38 \\
102 \\
256 \\
2 \\
136 \\
21 \\
59 \\
\text { n.d. } \\
3 \\
11 \\
26\end{array}$ & $\begin{array}{r}178 \\
450 \\
186 \\
6 \\
113 \\
28 \\
40 \\
2 \\
12 \\
12 \\
14\end{array}$ & $\begin{array}{c}148 \\
43 \\
550 \\
- \\
11 \\
95 \\
- \\
38 \\
\text { n.d. } \\
4 \\
47\end{array}$ & $\begin{array}{r}158 \\
47 \\
481 \\
197 \\
6 \\
109 \\
26 \\
46 \\
1 \\
- \\
19\end{array}$ & $\begin{array}{r}14 \\
15 \\
14 \\
120 \\
70 \\
672 \\
1198 \\
225 \\
16 \\
39 \\
70 \\
20\end{array}$ & $\begin{array}{r}263 \\
54 \\
340 \\
239 \\
50 \\
1382 \\
1200 \\
230 \\
105 \\
90 \\
161 \\
28\end{array}$ \\
\hline Y & $\mathrm{N}$ & & & $\mathrm{T}$ & & & & & $\mathrm{N}$ & & $\mathrm{N}$ & & & & & & $\mathrm{T}$ & & & \\
\hline $\begin{array}{l}\mathrm{Y} \\
\mathrm{La} \\
\mathrm{Ce} \\
\mathrm{Nd} \\
\mathrm{Sm} \\
\mathrm{Eu} \\
\mathrm{Gd} \\
\mathrm{Dy} \\
\mathrm{Er} \\
\mathrm{Yb} \\
\mathrm{Lu}\end{array}$ & $\begin{array}{c}20.9 \\
1.29 \\
3.17 \\
3.70 \\
1.51 \\
0.57 \\
2.14 \\
3.01 \\
1.74 \\
2.01 \\
0.29\end{array}$ & & & $\begin{array}{c}15.2 \\
1.10 \\
- \\
-\overline{11} \\
0.46 \\
1.87 \\
2.44 \\
1.60 \\
1.60 \\
0.25\end{array}$ & & & & & $\begin{array}{c}14.9 \\
1.24 \\
4.40 \\
4.46 \\
1.70 \\
0.67 \\
2.46 \\
2.53 \\
1.24 \\
1.38 \\
0.20\end{array}$ & & $\begin{array}{c}41.5 \\
3.60 \\
13.2 \\
12.6 \\
4.94 \\
1.53 \\
5.95 \\
6.93 \\
3.47 \\
3.69 \\
0.55\end{array}$ & & & & & & $\begin{array}{c}15.8 \\
- \\
3.50 \\
3.10 \\
1.62 \\
0.65 \\
2.46 \\
2.77 \\
1.73 \\
1.59 \\
0.24\end{array}$ & & & \\
\hline $\begin{array}{l}\mathrm{Ti} / \mathrm{Zr} \\
\mathrm{Ba} / \mathrm{Nb} \\
\mathrm{Zr} / \mathrm{Nb}\end{array}$ & $\begin{array}{r}193 \\
8 \\
11\end{array}$ & 183 & $\begin{array}{r}177 \\
12 \\
12\end{array}$ & 150 & $\begin{array}{r}225 \\
25 \\
23\end{array}$ & $\begin{array}{l}16 \\
10 \\
44\end{array}$ & $\begin{array}{r}121 \\
37 \\
32\end{array}$ & $\begin{array}{r}120 \\
18 \\
41\end{array}$ & $\begin{array}{r}118 \\
17 \\
21\end{array}$ & 109 & $\begin{array}{l}67 \\
11 \\
64\end{array}$ & $\begin{array}{r}93 \\
3 \\
50\end{array}$ & $\begin{array}{r}97 \\
2 \\
38\end{array}$ & $\begin{array}{r}75 \\
7 \\
36\end{array}$ & 107 & $\begin{array}{r}124 \\
16 \\
23\end{array}$ & 127 & 107 & & \\
\hline $\begin{array}{l}{ }^{87} \mathrm{Sr} / 86 \\
{ }^{143} \mathrm{Nd} /{ }^{144} \\
{ }^{206} \mathrm{~Pb} /{ }^{104} \mathrm{Nd} \\
{ }^{207} \mathrm{~Pb} /{ }^{04} \mathrm{~Pb} \\
{ }^{208} \mathrm{~Pb} /{ }^{044} \mathrm{~Pb}\end{array}$ & $\begin{array}{l}18.61 \\
15.56 \\
38.33\end{array}$ & $\begin{array}{l}615(3) \\
8(7) \\
8(7) \\
9(18)\end{array}$ & & & & & & & $\begin{array}{r}0.704 \\
0.513 \\
18.613 \\
15.565 \\
38.344\end{array}$ & $\begin{array}{l}30(4) \\
23(2) \\
(5) \\
(5) \\
(15)\end{array}$ & $\begin{array}{r}0.7048 \\
0.5132 \\
18.561 \\
15.517 \\
38.141 \\
38\end{array}$ & $\begin{array}{l}88(3) \\
24(5) \\
(6) \\
(6) \\
(16)\end{array}$ & & & & & & & & \\
\hline
\end{tabular}

C Nancy, France). Y analyses carried out by ICP are reported with decimals. Precision for isotopic data, expressed as 2 sigma, is reported in brackets. $\mathrm{LOI}=\operatorname{loss}$ on ignition; $\mathrm{mgv}=\mathrm{Mg} /\left(\mathrm{Mg}+\mathrm{Fe}^{2+}\right) ;-=$ not determined; $\mathrm{n} . \mathrm{d}$. $=$ not detected. Analyses of reference standards $\mathrm{AGV} 1$ and $\mathrm{BR}$ are 

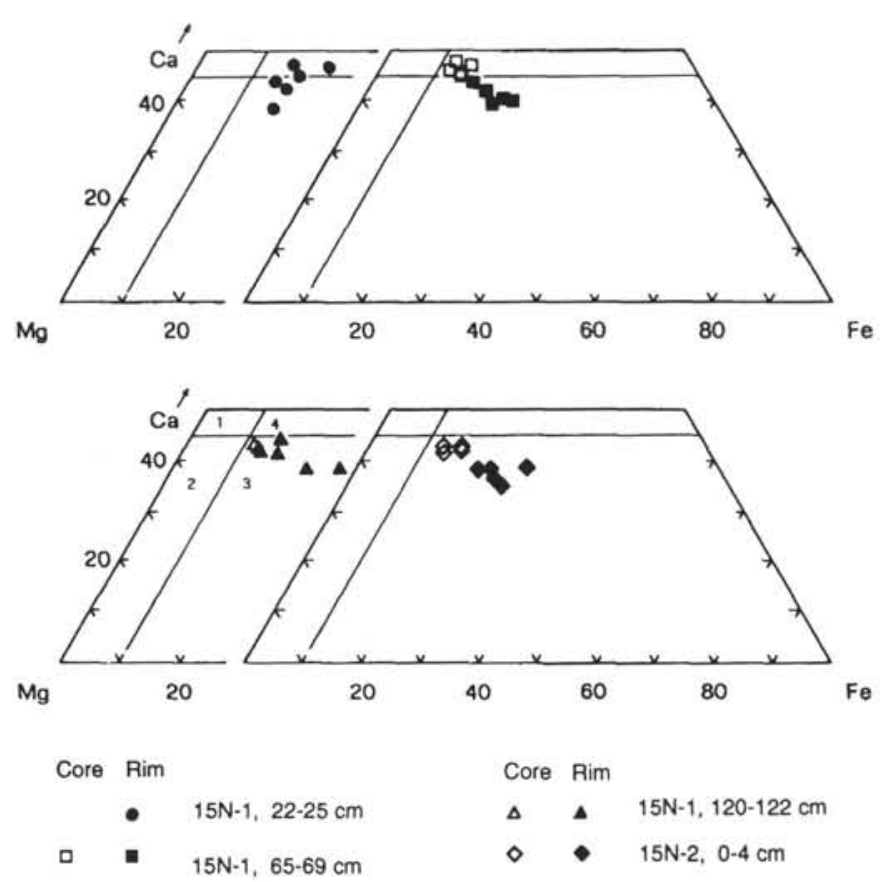

Figure 2. Clinopyroxene compositions of igneous rocks recovered from Site 828 in the $\mathrm{Ca}-\mathrm{Mg}-\left(\mathrm{Fe}_{1}+\mathrm{Mn}\right)$ diagram. The numbers $1,2,3$, and 4 indicate diopside, endiopside, augite, and salite fields, respectively.

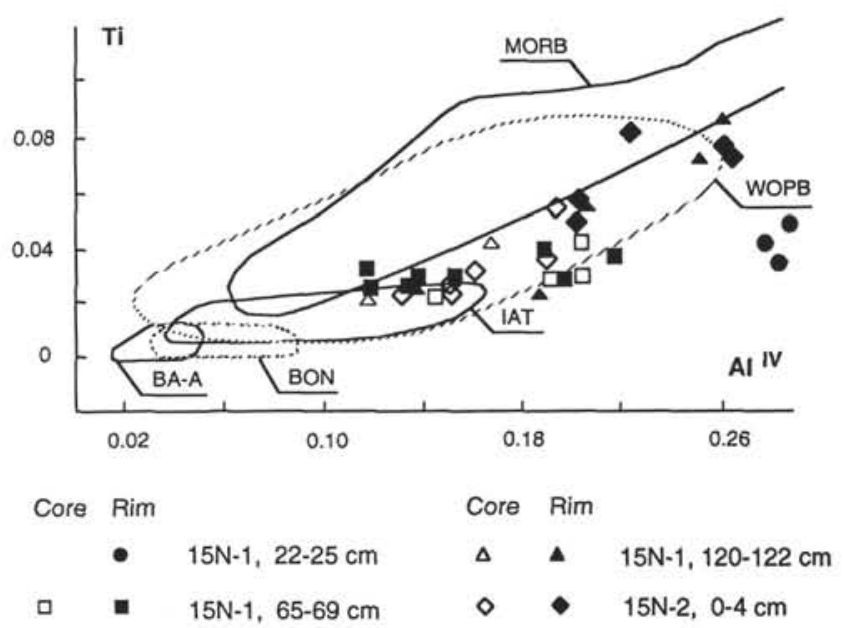

Figure 3. Ti vs. $\mathrm{Al}^{\mathrm{IV}}$ contents (at\%) in clinopyroxenes from basaltic rocks of Hole 828A. Fields of mid-ocean ridge basalts (MORB), within-oceanic plate basalts (WOPB), island arc tholeiites (IAT), boninites (BON), and basaltic andesites and andesites (BA-A) from the forearc region are from Beccaluva et al. (1989). Composition of microphenocrysts and groundmass crystals are indicated as core and rim respectively.

Primary mineralogy and texture have been used to distinguish four main groups of basalts and one rhyolitic group, which are discussed below:

1. Aphyric to sparsely plagioclase + clinopyroxene-phyric basalts. These basalts have lath-shaped plagioclase and rounded clinopyroxene microphenocrysts as isolated or glomeroporphyritic aggregates. Clinopyroxene is frequently hourglass-zoned. The groundmass is intergranular, fine- to medium-grained, and comprised of skeletal plagioclase microlites with small clinopyroxenes, opaque phases and glass. Where glass is dominant, textures fade through hyalopilitic to almost aphyric. Opaque minerals occur in irregular patches, or replace mafic phases. The rare veins and fractures are infilled with microcrystalline calcite, clay minerals and oxides.

2. Moderately olivine + plagioclase-phyric basalts. Euhedral plagioclase $(2 \%-3 \%)$, partially replaced by clay minerals, and olivine $(<1 \%)$ pseudomorphed by serpentine, constitute the phenocrysts. The groundmass is intersertal in texture, with tiny microlites of plagioclase and small clinopyroxene and oxide interstitial grains. In places, textures become hyalopilitic with abundant, partially devitrified glass between plagioclase microlites. Crosscutting veins and fractures are filled by calcite in their cores and zeolites and smectites at their margins, with the thinnest veins containing microcrystalline calcite, oxides and clay minerals.

3. Highly olivine + plagioclase phyric basalts. In this group, large olivine crystals (15\%) up to $2 \mathrm{~mm}$ across, which are totally pseudomorphed by serpentine and minor calcite, occur with highly altered plagioclase laths (5\% and up to $2-3 \mathrm{~mm}$ ). The latter often have narrow, fresh rims. Intergranular to hyalopilitic groundmass contains skeletal, $\mathrm{H}$-shaped plagioclase microlites intergrown with acicular clinopyroxenes in a quench texture, set in altered glass. In some samples (e.g., Sample 134-828A-15N-1, 20-22 cm), the phenocrysts are rimmed by opaque minerals, and altered, partially devitrified brown glass constitutes the entire groundmass. Spherical or lobate vesicles $(-20 \%)$ are filled with radiating fibrous calcite with a "cockade" texture.

4. Dolerites. Samples with doleritic textures occur close to the bottom of Hole $828 \mathrm{~B}$. They are holocrystalline, medium-grained with idiomorphic plagioclase laths slightly opacized by clay minerals, and interstitial subhedral clinopyroxene and opaque minerals. Extremely rare olivine microphenocrysts are totally pseudomorphed by iddingsite. Chlorite is widespread, both replacing primary mafic phases, and as a dispersed secondary mineral.

5. Rhyolite. This rock type is represented by a single porphyritic clast (Sample 134-828B-2R-CC, 3-6 cm) in which euhedral, cloudy, albitic plagioclase and deformed, embayed, quartz phenocrysts are set in a fine-grained groundmass composed of quartz and alkali feldspar. Chlorite and epidote, together with minor oxides, replace a rare femic phase (biotite?).

\section{MINERAL CHEMISTRY}

The petrographic descriptions above indicate that the basaltic rocks from Site 828 were strongly altered via rock-sea water interaction. Few minerals have preserved original magmatic compositions. No olivine relics were found. Occasional groundmass plagioclases have compositions around $\mathrm{An}_{70-72}$, whereas all others are almost pure albite. Several small grains of spinel and magnetite were analyzed in Samples 134-828A-15N-1, 22-25 cm, and -15N-1, 120-122 cm, respectively. Spinels have $\mathrm{Cr}_{2} \mathrm{O}_{3}$ contents varying from 38.0 to 39.4 $\mathrm{wt} \%$, and $\mathrm{mgv}\left(\mathrm{mgv}=\mathrm{Mg} /\left[\mathrm{Mg}+\mathrm{Fe}^{2+}\right]\right)$ values around 0.67 . Neither $\mathrm{TiO}_{2}$ contents $(0.24-0.56)$, nor $\mathrm{Cr} /(\mathrm{Cr}+\mathrm{Al})$ values of spinels can effectively discriminate between MORB and back-arc basin basalt (BABB) magmatic affinity (Dick and Bullen, 1984). Magnetites have low $\mathrm{TiO}_{2}$ contents $(0.26-1.28 \mathrm{wt} \%)$.

Clinopyroxenes are the only phase that has been largely preserved. Representative compositions are reported in Table 2, and all analyzed grains are plotted in the pyroxene quadrilaterals in Figure 2. All clinopyroxenes fall in the salite to augite fields and are normally zoned. Those from Sample 134-828A-15N-1, 22-25 cm, plot in a restricted area and are characterized by lower $\mathrm{SiO}_{2}$ and higher $\mathrm{Al}_{2} \mathrm{O}_{3}$ contents compared to other clinopyroxenes. In the $\mathrm{Ti}$ vs. $\mathrm{Al}^{\mathrm{IV}}$ diagram (Beccaluva et al., 1989), clinopyroxenes from this sample plot to the right of the MORB pyroxene trend, whereas the other samples parallel the MORB trend, but at lower Ti contents (Fig. 3).

\section{WHOLEROCK GEOCHEMISTRY}

Major and trace element analyses of basaltic samples recovered from $\mathrm{Site} 828$, together with $\mathrm{Sr}, \mathrm{Nd}$ and $\mathrm{Pb}$ isotopic ratios, are reported 
Table 2. Representative clinopyroxene compositions of basaltic rocks recovered from Hole 828A.

\begin{tabular}{|c|c|c|c|c|c|c|c|c|c|c|c|c|c|c|c|c|}
\hline \multirow[t]{2}{*}{$\begin{array}{l}\text { Core, section: } \\
\text { Interval }(\mathrm{cm}) \text { : }\end{array}$} & \multicolumn{3}{|c|}{$\begin{array}{l}15 \mathrm{~N}-1 \\
22-25 \\
\end{array}$} & \multicolumn{5}{|c|}{$\begin{array}{l}15 \mathrm{~N}-1 \\
65-69\end{array}$} & \multicolumn{4}{|c|}{$\begin{array}{c}15 \mathrm{~N}-1 \\
120-122\end{array}$} & \multicolumn{4}{|c|}{$\begin{array}{c}5 \mathrm{~N}-2 \\
0-4\end{array}$} \\
\hline & $\mathrm{gm}$ & $\mathrm{gm}$ & $\mathrm{gm}$ & A ph c & A ph r & B ph c & B ph r & $\mathrm{C}_{\mathrm{ph} \mathrm{c}}$ & A ph c & A ph i & A ph r & $\mathrm{gm}$ & A ph c & A ph r & B ph c & $\mathrm{gm}$ \\
\hline $\mathrm{SiO}_{2}$ & 46.22 & 44.66 & 46.40 & 48.64 & 49.07 & 48.56 & 48.76 & 50.07 & 49.44 & 51.46 & 48.22 & 45.42 & 48.62 & 48.12 & 48.08 & 45.61 \\
\hline $\mathrm{TiO}_{2}$ & 1.43 & 0.97 & 1.25 & 1.00 & 1.12 & 1.08 & 0.93 & 0.79 & 1.44 & 0.77 & 1.92 & 2.88 & 1.37 & 1.71 & 1.98 & 2.57 \\
\hline $\mathrm{Al}_{2} \mathrm{O}_{3}$ & 7.97 & 12.31 & 9.67 & 6.46 & 2.84 & 6.10 & 3.39 & 3.76 & 4.16 & 2.70 & 5.15 & 5.37 & 4.15 & 4.75 & 5.00 & 5.50 \\
\hline $\mathrm{Fe}_{2} \mathrm{O}_{3}$ & 4.43 & 3.39 & 2.92 & 1.95 & 1.98 & 3.35 & 2.70 & 3.18 & 2.83 & 2.75 & 3.31 & 4.48 & 4.53 & 3.84 & 2.83 & 4.94 \\
\hline $\mathrm{FeO}$ & 6.03 & 5.84 & 7.19 & 5.79 & 13.09 & 4.72 & 11.81 & $\begin{array}{l}5.169 \\
5.69\end{array}$ & 7.33 & 4.93 & $\begin{array}{l}6.18 \\
6.18\end{array}$ & $\begin{array}{r}4.40 \\
12.50\end{array}$ & 5.11 & 8.75 & 6.95 & $\begin{array}{l}4.94 \\
10.58\end{array}$ \\
\hline $\mathrm{MnO}$ & 0.15 & 0.21 & 0.17 & 0.20 & 0.41 & 0.19 & 0.34 & 0.27 & 0.22 & 0.19 & 0.22 & 0.37 & 0.21 & 0.36 & 0.22 & $0.33^{\circ}$ \\
\hline $\mathrm{MgO}$ & 14.70 & 11.45 & 12.97 & 13.18 & 11.61 & 13.51 & 11.91 & $\begin{array}{r}14.39 \\
\end{array}$ & 14.41 & 15.96 & 13.68 & 10.89 & 14.42 & 13.94 & 14.02 & 12.75 \\
\hline $\mathrm{CaO}$ & 18.06 & 20.92 & 19.36 & 22.28 & 19.03 & 22.50 & 19.17 & 21.99 & 20.16 & 21.36 & 21.09 & $\begin{array}{l}17.69 \\
\end{array}$ & 20.91 & 18.57 & 20.06 & 16.83 \\
\hline $\mathrm{Na}_{2} \mathrm{O}$ & 0.22 & 0.22 & 0.29 & 0.22 & 0.24 & 0.26 & 0.26 & 0.17 & 0.29 & 0.28 & 0.34 & 0.42 & 0.33 & 0.28 & 0.30 & 0.36 \\
\hline $\mathrm{Cr}_{2} \mathrm{O}_{3}$ & 0.06 & 0.02 & 0.05 & 0.06 & 0.00 & 0.13 & 0.02 & 0.09 & 0.02 & 0.40 & 0.00 & 0.03 & 0.09 & 0.01 & 0.12 & 0.00 \\
\hline Total & 99.29 & 99.98 & 100.28 & 99.79 & 99.40 & 100.39 & 99.29 & 100.40 & 100.30 & 100.80 & 100.11 & 100.05 & 99.74 & 100.33 & 99.56 & 99.47 \\
\hline $\mathrm{Si}$ & 1.720 & 1.652 & 1.713 & 1.810 & 1.884 & 1.798 & 1.867 & 1.856 & 1.832 & 1.881 & 1.792 & 1.737 & 1.810 & 1.795 & 1.795 & 1.736 \\
\hline $\mathrm{Ti}$ & 0.041 & 0.027 & 0.035 & 0.028 & 0.032 & 0.030 & 0.027 & 0.022 & 0.041 & 0.022 & 0.054 & 0.084 & 0.039 & 0.049 & 0.057 & 0.075 \\
\hline $\mathrm{Al}$ & 0.361 & 0.555 & 0.435 & 0.283 & 0.129 & 0.266 & 0.153 & 0.164 & 0.188 & 0.120 & 0.233 & 0.250 & 0.188 & 0.216 & 0.228 & 0.255 \\
\hline $\mathrm{Fe}^{3+}$ & 0.126 & 0.095 & 0.082 & 0.055 & 0.057 & 0.093 & 0.078 & 0.089 & 0.080 & 0.076 & 0.094 & 0.130 & 0.129 & 0.109 & 0.080 & 0.143 \\
\hline $\mathrm{Fe}^{2+}$ & 0.190 & 0.183 & 0.225 & 0.180 & 0.420 & 0.146 & 0.378 & 0.177 & 0.230 & 0.153 & 0.194 & 0.405 & 0.161 & 0.277 & 0.220 & 0.341 \\
\hline $\mathrm{Mn}$ & 0.005 & 0.007 & 0.006 & 0.006 & 0.013 & 0.006 & 0.011 & 0.009 & 0.007 & 0.006 & 0.007 & 0.012 & 0.007 & 0.012 & 0.007 & 0.011 \\
\hline $\mathrm{Mg}$ & 0.823 & 0.638 & 0.721 & 0.731 & 0.664 & 0.746 & 0.680 & 0.765 & 0.804 & 0.878 & 0.765 & 0.627 & 0.808 & 0.783 & 0.788 & 0.730 \\
\hline $\mathrm{Ca}$ & 0.719 & 0.829 & 0.765 & 0.889 & 0.783 & 0.893 & 0.787 & 0.873 & 0.800 & 0.836 & 0.839 & 0.724 & 0.833 & 0.741 & 0.802 & 0.685 \\
\hline $\mathrm{Na}$ & 0.017 & 0.016 & 0.021 & 0.016 & 0.018 & 0.019 & 0.019 & 0.012 & 0.022 & 0.021 & 0.025 & 0.032 & 0.025 & 0.021 & 0.023 & 0.027 \\
\hline $\mathrm{Cr}$ & 0.002 & 0.001 & 0.002 & 0.002 & 0.000 & 0.004 & 0.001 & 0.003 & 0.001 & 0.012 & 0.000 & 0.001 & 0.003 & 0.000 & 0.004 & 0.000 \\
\hline Total & 4.003 & 4.003 & 4.003 & 4.000 & 4.000 & 4.000 & 4.000 & 4.000 & 4.004 & 4.004 & 4.003 & 4.004 & 4.004 & 4.004 & 4.004 & 4.004 \\
\hline $\mathrm{mgv}$ & 0.81 & 0.78 & 0.76 & 0.80 & 0.61 & 0.84 & 0.64 & 0.81 & 0.78 & 0.85 & 0.80 & 0.61 & 0.83 & 0.74 & 0.78 & 0.68 \\
\hline Wo & 38.6 & 47.3 & 42.5 & 47.8 & 40.4 & 47.4 & 40.7 & 45.7 & 41.6 & 42.9 & 44.2 & 38.1 & 43.0 & 38.6 & 42.3 & 35.9 \\
\hline En & 44.2 & 36.4 & 40.1 & 39.3 & 34.3 & 39.6 & 35.2 & 40.0 & 41.9 & 45.0 & 40.3 & 33.0 & 41.7 & 40.7 & 41.5 & 38.2 \\
\hline $\mathrm{Fe}$ & 17.2 & 16.3 & 17.4 & 13.0 & 25.3 & 13.0 & 24.2 & 14.3 & 16.5 & 12.1 & 15.5 & 28.8 & 15.3 & 20.7 & 16.2 & 25.9 \\
\hline
\end{tabular}

Notes: $\mathrm{mgv}=\mathrm{Mg} / \mathrm{Mg}+\mathrm{Fe}(\mathrm{at} \%) ; \mathrm{Wo}$, En, and Fs indicate wollastonite enstatite, and ferrosilite percentages. Abbreviations: $\mathrm{ph}=$ phenocrysts; $\mathrm{mph}=$ microphenocryst; gm = groundmass; $\mathrm{c}=$ core; $\mathrm{r}=$ rim. $\mathrm{A}, \mathrm{B}$, and $\mathrm{C}$ indicate different crystals. Atoms for formula units are calculated on the base of 6 oxygens. 


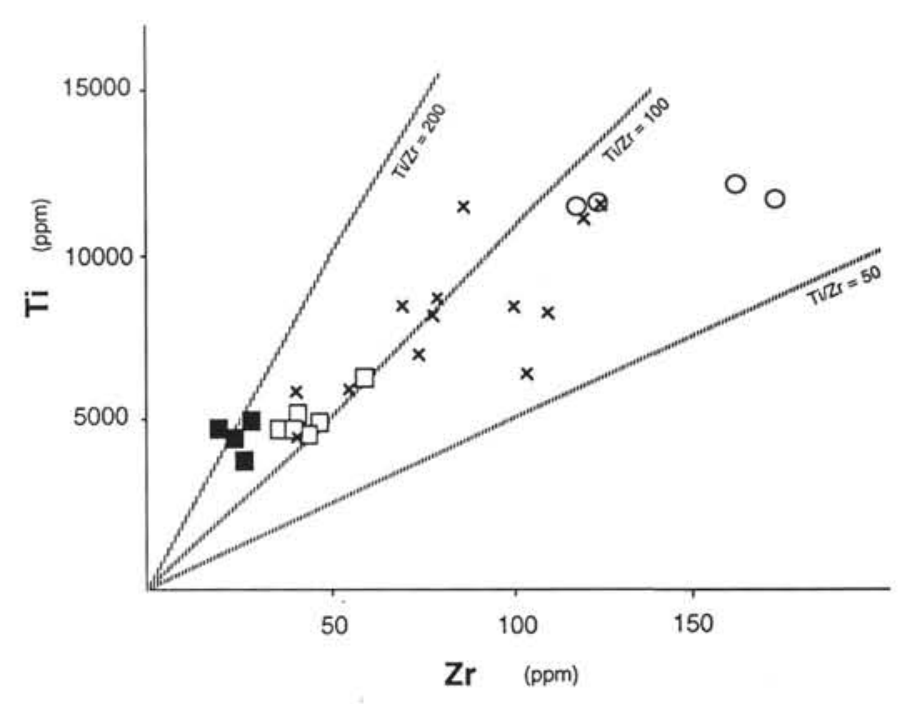

Figure 4. $\mathrm{Ti}$ vs. $\mathrm{Zr}$ contents in basaltic rocks recovered from Site 828. Open circles $=$ MORB ; open squares $=$ Pic-BABB; filled squares $=$ BABB; crosses $=$ basaltic rocks dredged from DEZ by Maillet et al. (1983) and Collot et al. (1992).

in Table 1. Due to the high degree of alteration of these samples, their chemistry must be interpreted with care. Seafloor weathering effects are particularly reflected in the high LOI values, between 2.6 and $9.6 \mathrm{wt} \%$ (except for two samples that have LOI $<2 \mathrm{wt} \%$ ). Alteration and the occurrence of secondary calcite filling veinlets and vugs result in anomalously high $\mathrm{CaO}$ (up to $20 \mathrm{wt} \%$ ), coupled with low $\mathrm{SiO}_{2}$ contents.

On the basis of major element geochemistry, the volcanic rocks from Holes $828 \mathrm{~A}$ and $828 \mathrm{~B}$ can be classified as basalts $\left(\mathrm{SiO}_{2}<53\right.$ wt\%); a rhyolite (Sample 134-828B-2R-CC, 3-6 cm) and a dolerite (Sample 134-828B-3R-CC, 6-7 cm) were also recovered. At least two compositional groups of samples can be identified: Group A is characterized by $\mathrm{TiO}_{2}$ content less than $1.05 \mathrm{wt} \%$ (usually around 0.7 wt $\%), \mathrm{Al}_{2} \mathrm{O}_{3}$ between $14.9 \%$ and $18.2 \%$, and $\mathrm{Na}_{2} \mathrm{O}$ ranging from $2.99 \%$ to $5.03 \%$. Some lavas of this group are notable for their picritic tendency, with mgv values reaching $0.80, \mathrm{MgO}$ up to $14.9 \mathrm{wt} \%$, and $\mathrm{Ni}$ up to $190 \mathrm{ppm}$. Group $\mathrm{B}$, on the other hand, has $\mathrm{TiO}_{2}$ content around $2 \%(1.9 \%-2.0 \%), \mathrm{Al}_{2} \mathrm{O}_{3}$ consistently lower than $15 \%$, and $\mathrm{Na}_{2} \mathrm{O}$ greater than $5 \%$. Samples within this group are more evolved than Group A, with mgv always $<0.6, \mathrm{MgO}$ content between 6.7 and $8.1 \mathrm{wt} \%$, and $\mathrm{Ni}$ from 112 to $76 \mathrm{ppm}$.

The two groups are well separated on a $\mathrm{Ti}-\mathrm{Zr}$ discrimination diagram (Fig. 4); Ti/Zr values of Group B are always $<100$ (67-97), whereas Group A has values ranging from 107 to 225 . Other trace element ratios, such as $\mathrm{Zr} / \mathrm{Nb}, \mathrm{Zr} / \mathrm{Y}, \mathrm{Ba} / \mathrm{Zr}$, or $\mathrm{Sr} / \mathrm{Zr}$ also clearly distinguish between these basaltic groups, with only minimal overlap (Table 1), although $\mathrm{Ba}$ and $\mathrm{Sr}$ may have been affected by secondary mobilization.

The MORB-normalized incompatible element patterns (Fig. 5) permit a more comprehensive comparison of the basalt geochemistry, although elements on the left side of these diagrams are susceptible to secondary mobilization (Philpotts et al., 1969; Alt et al., 1986; Bienvenu et al., 1990). Three diagrams are drawn, two for group A (Figs. $5 \mathrm{~A}$ and B) and one for group B (Fig. 5C). Samples 134-828A-15N-1, $119-120 \mathrm{~cm},-15 \mathrm{~N}-1,122-123 \mathrm{~cm},-15 \mathrm{~N}-2,3-4 \mathrm{~cm}$, and $-15 \mathrm{~N}-2,7-9$ $\mathrm{cm}$ (Fig. $5 \mathrm{C}$ ) have almost flat patterns close to $1 \times \mathrm{N}-\mathrm{MORB}$ abundances, except for $\mathrm{K}, \mathrm{Rb}$, and sometimes $\mathrm{Ba}$. This, together with the geochemical features noted above, indicates a MORB affinity for these rocks. In contrast, lavas of Group A (Figs. 5A and B) are depleted relative to $\mathrm{N}-\mathrm{MORB}$ in the elements from $\mathrm{Nb}$ through $\mathrm{Y}$, and enriched in $\mathrm{K}, \mathrm{Rb}$, and $\mathrm{Ba}$. In this respect, the distribution of high field strength elements (HFSE), considered immobile during rock-seawater interaction (Mottl and Holland, 1978), is of particular interest in identifying
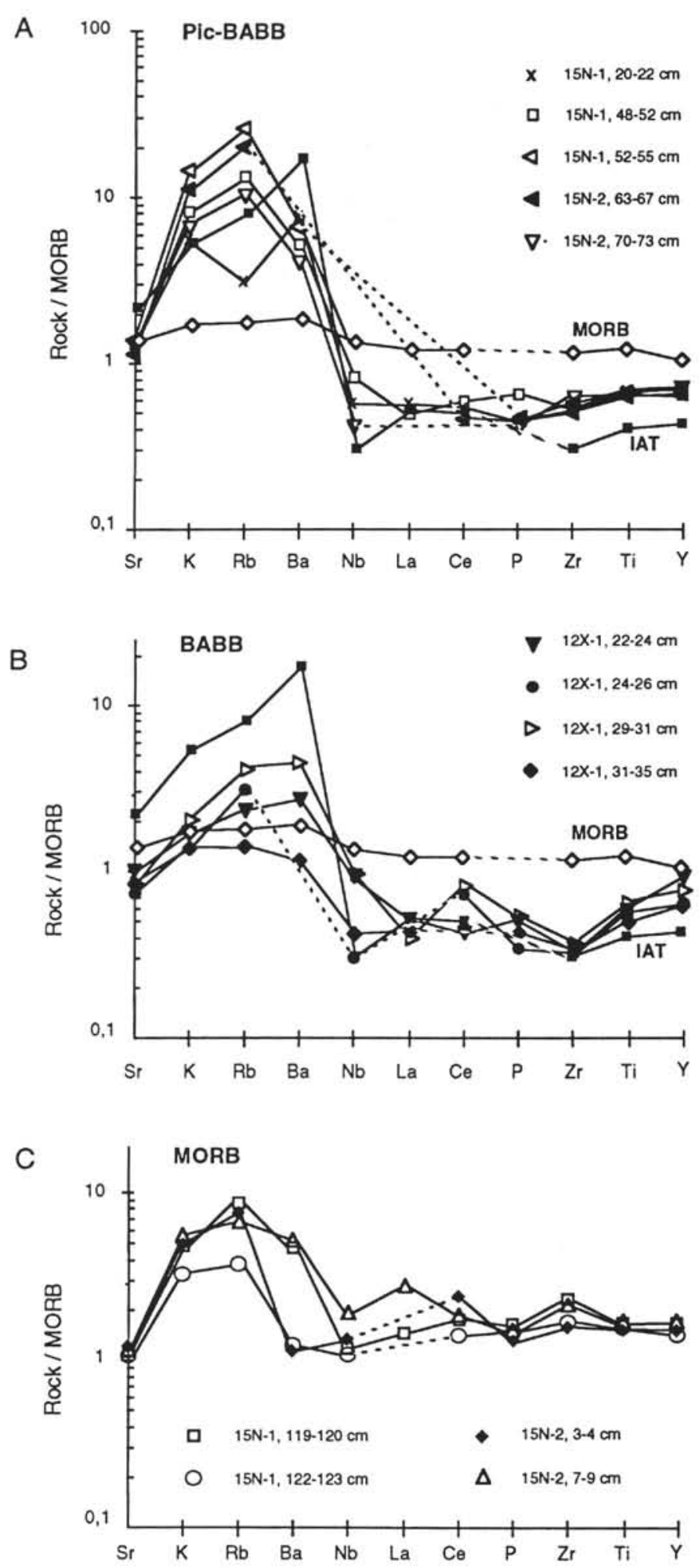

Figure 5. MORB-normalized incompatible element patterns for the three groups of basaltic rocks recovered from Site 828. Patterns of MORB and IAT are from Sun (1980). Normalizing values from Sun and McDonough (1989).

magmatic affinities. The picritic lavas plot around $0.5 \times$ MORB for $\operatorname{HFSE}(\mathrm{Nb}$ to $\mathrm{Y}$ ), and between 2 and $20 \times$ MORB for low-field strength element (LFSE) (except Sr). The other rocks of this group (Fig. 5B), apart from a slight scatter for $\mathrm{Ce}$, have HFSE levels between 0.3 and 1 $\times$ MORB levels. The presence of a negative $\mathrm{Zr}$ anomaly is a peculiar characteristic of these rocks. The marked depletion in HFSE of sam- 
ples in Group A is atypical of MORB magmas, but similar to the well-documented HFSE depletions in lavas associated with subduction, including IAT and many, but not all, BABB.

The chondrite-normalized REE patterns of the two different groups (Fig. 6) permit a clear distinction between MORB (Group B) and MORB/IAT transitional type lavas (Group A). Sample 134-828A$15 \mathrm{~N}-1,119-120 \mathrm{~cm}$, has a light rare earth element (LREE) depleted pattern $\left([\mathrm{La} / \mathrm{Yb}]_{\mathrm{N}}=0.70\right)$ typical of normal MORB (from 10 to $20 \times$ chondritic), whereas Group A lavas have even more LREE-depleted patterns $\left([\mathrm{La} / \mathrm{Yb}]_{\mathrm{N}}=0.46-0.64\right)$, and overall lower REE abundances, generally less than $8 \times$ chondritic.

${ }^{87} \mathrm{Sr} /{ }^{86} \mathrm{Sr}$ ratios for Group A and B lavas are unusually high (0.7043-0.7062) for both MORB and intra-oceanic island arc volcanism. This is probably due to rock-seawater interaction, which commonly increases radiogenic strontium (Hart et al., 1974; Menzies and Seyfried, 1979; Brown et al., 1989). ${ }^{143} \mathrm{Nd} /{ }^{144} \mathrm{Nd}$ isotopic ratios are around 0.5132 and are among the highest values observed for ocean floor basalts worldwide $(0.5130-0.5132$ for the Pacific and Indian oceans; $0.5130-0.5133$ for the Atlantic Ocean; Wilson, 1989). ${ }^{207} \mathrm{~Pb} /$ ${ }^{204} \mathrm{~Pb}$ vs. ${ }^{206} \mathrm{~Pb} /{ }^{204} \mathrm{~Pb}$ isotopic ratios are reported in Table 1 and plotted in Figure 7. In terms of ${ }^{207} \mathrm{~Pb} /{ }^{204} \mathrm{~Pb}$ vs. ${ }^{206} \mathrm{~Pb} /{ }^{204} \mathrm{~Pb}$, Sample $134-828 \mathrm{~A}-$ $15 \mathrm{~N}-1,119-120 \mathrm{~cm}$, with normal-MORB affinity, plots within the field of Pacific and Indian MORBs (not distinguished on the figure). On the other hand, Group A lavas, being slightly enriched in radiogenic lead, plot outside the MORB field.

\section{DISCUSSION}

Three groups of rocks with different magmatic affinity have been distinguished among volcanics recovered from the flank of NDR. Group B lavas are sparsely plagioclase + clinopyroxene-phyric basalts, with $\mathrm{TiO}_{2}$ content around $2 \mathrm{wt} \%$ and $\mathrm{Al}_{2} \mathrm{O}_{3}$ consistently lower than 15 $\mathrm{wt} \%$. In terms of major element contents they match normal-MORB compositions. They have flat MORB-normalized incompatible element patterns and LREE-depleted chondrite-normalized REE pat-

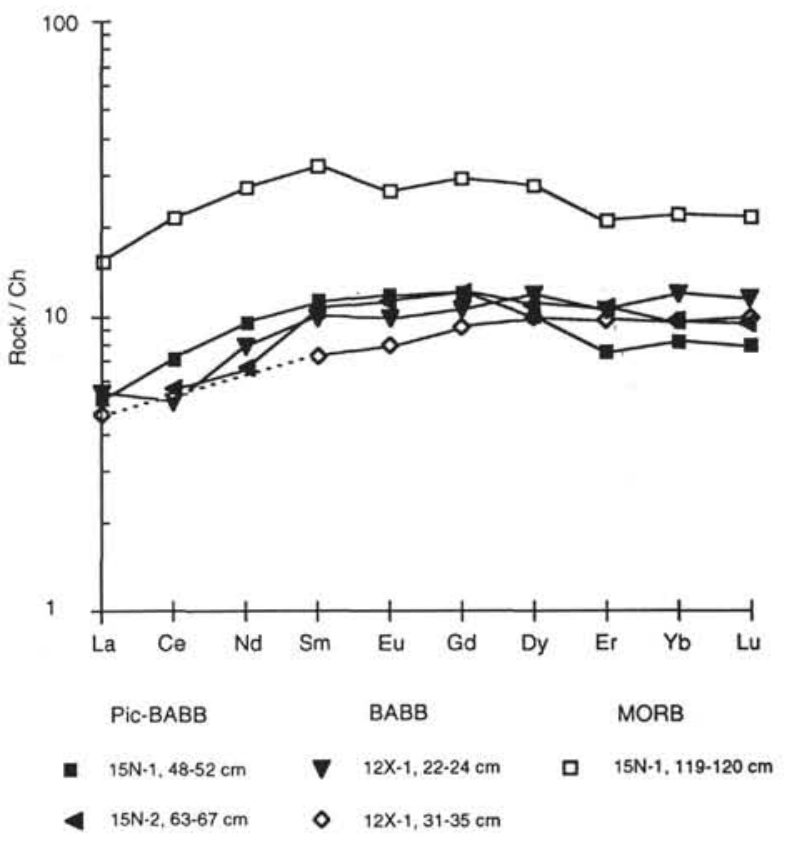

Figure 6. Chondrite-normalized REE distributions for the three groups of basaltic rocks recovered from Site 828. Normalizing values from Sun and McDonough (1989).

terns. Their fairly low mgv values $(0.53-0.59)$, together with a weak negative Eu anomaly $\left(\mathrm{Eu} / \mathrm{Eu}^{*}=0.86 ; \mathrm{Eu} / \mathrm{Eu}^{*}=\mathrm{Eu}_{\mathrm{N}} /\left[\mathrm{Sm}_{\mathrm{N}}+\mathrm{Gd}_{\mathrm{N}}\right] / 2\right)$, suggest that such magmas underwent extensive plagioclase plus olivine fractionation. Taking a $\mathrm{Zr}$ content of $89 \mathrm{ppm}$ for primary MORB magmas (N2 MORB; Viereck et al., 1989), a simple Rayleigh fractionation model indicates that they could correspond to liquid fraction

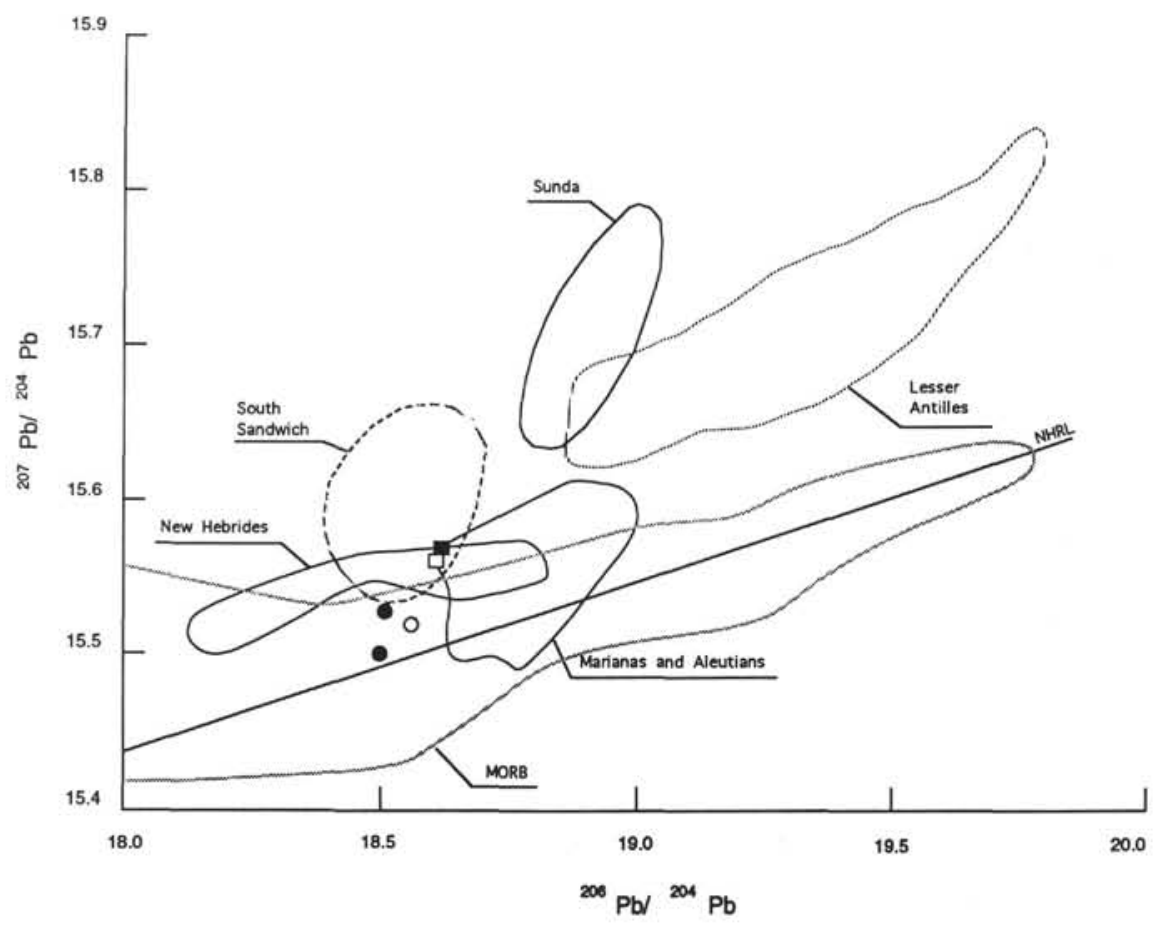

Figure 7. ${ }^{207} \mathrm{~Pb} /{ }^{204} \mathrm{~Pb}$ vs. ${ }^{206} \mathrm{~Pb} /{ }^{204} \mathrm{~Pb}$ isotopic ratios of basaltic rocks recovered from Site 828 . Filled square $=$ BABB ; open square $=$ Pic - BABB $;$ open circle $=$ MORB; solid circles $=$ Site 831 basalts. Field of basaltic rocks from island arc and mid-ocean ridge settings are from Sun (1980), White and Dupré (1986), and Wilson (1989). New Hebrides Island Arc field from Briqueu et al. (this volume). NHRL = North Hemisphere Reference Line. 


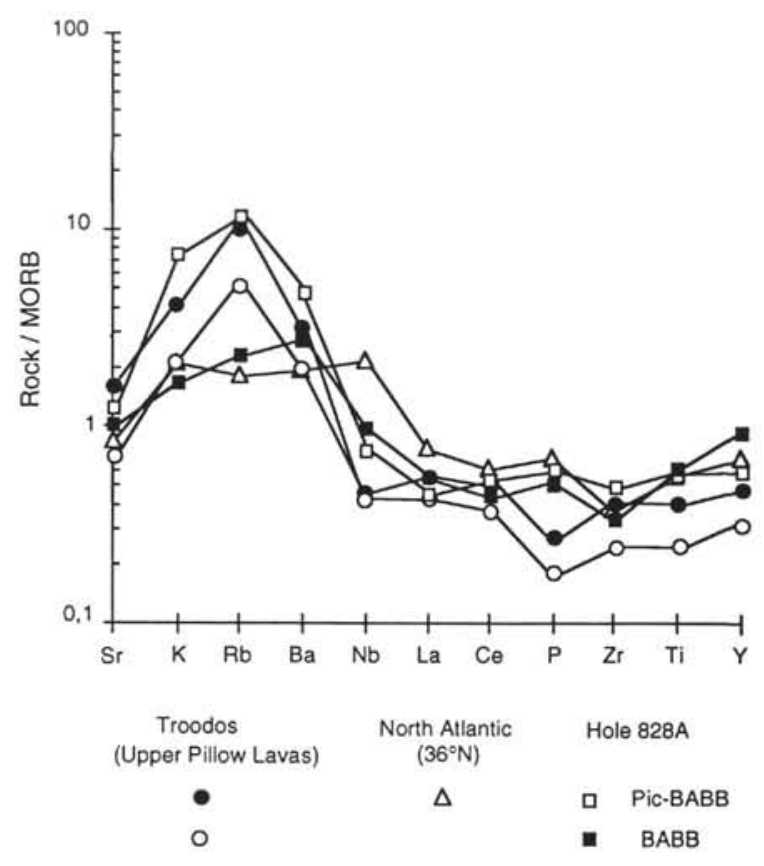

Figure 8. MORB-normalized incompatible element patterns of BABB and P-BABB lavas from Site 828 compared with picritic MORB (Wood et al., 1979) and upper pillow lavas of the Troodos ophiolitic complex (Cameron, 1985).

between 0.76 and 0.51 . In summary, petrographical features, major and trace element geochemistry, inter-elemental ratios such as $\mathrm{Ti} / \mathrm{Zr}$, $\mathrm{Zr} / \mathrm{Nb}, \mathrm{Zr} / \mathrm{Y}$, and the only available isotopic determinations point to a normal MORB magmatic affinity for these rocks.

Group A lavas are moderately (Group A1) to highly (Group A2) olivine + plagioclase-phyric basalts, the latter showing a picritic tendency. Their magmatic affinity and geotectonic setting is debatable. Both basalt types are characterized by variable enrichment in LFSE and pronounced $\mathrm{HFSE}$ depletion, together with higher $\mathrm{Al}_{2} \mathrm{O}_{3}$ and lower $\mathrm{FeO}$ (at the same $\mathrm{MgO}$ ) contents with respect to MORB-type lavas.

Picrites from mid-ocean ridges usually have lower LFSE (when the effect of secondary alteration can be ruled out), slightly higher HFSE, and significantly higher Nb contents (Wood et al., 1979; Sun et al., 1979; Sun, 1980) than Group A2 picrites (Figs. 5 and 8). Some basaltic rocks from the Troodos ophiolitic complex have petrographic and geochemical features comparable to these NDR rocks. In fact Group I and II basalts of upper pillow lavas, despite somewhat lower $\mathrm{TiO}_{2}$ contents $(0.30 \%-0.60 \%)$, have $\mathrm{Ti} / \mathrm{Zr}(100-120), \mathrm{Zr} / \mathrm{Y}(2.2-$ $2.0), \mathrm{Zr} / \mathrm{Nb}(18-35)$ and $\mathrm{Y} / \mathrm{Nb}(9-15)$ values (Cameron, 1985), and MORB-normalized incompatible element patterns, which resemble Site 828 picritic basalts (Fig. 8, Table 1). Cameron (1985) concludes that these Troodos lavas have arc tholeiitic affinities. Because of the intermediate MORB/IAT compositional characteristics and the high modal olivine percentages (i.e., high $\mathrm{MgO}$ contents) of Group A2 rocks, we refer to them as picritic back-arc basin basalts (Pic-BABB).

A comparison of Group A1 basaltic rocks can be made with backarc basin basalts (BABB) from the North Fiji, Mariana, Lau and Sulu Sea back-arc basins, many of which show MORB/IAT transitional magmatic affinities (Hawkins and Melchior, 1985; Sinton and Fryer, 1987; Volpe, Macdougall, Hawkins, 1988; Price et al., 1990; Spadea et al., 1991). The main petrological characteristics of these lavas have recently been summarized by Falloon et al. (1992). Compared to MORB, BABB have higher $\mathrm{Al}_{2} \mathrm{O}_{3}$ and $\mathrm{Na}_{2} \mathrm{O}$ and lower $\mathrm{TiO}_{2}$ and $\mathrm{FeO}$ contents. They are also relatively enriched in LFSE, LREE, and $\mathrm{H}_{2} \mathrm{O}$ (Hawkins and Melchior, 1985; Sinton and Fryer, 1987), and they have more radiogenic $\mathrm{Sr}$ and $\mathrm{Pb}$ isotopic values (Volpe, Macdougall, Hawkins, 1988; Volpe, Macdougall, Lugmair, 1988). Site 828 Group A lavas of both types show higher $\mathrm{Al}_{2} \mathrm{O}_{3}$ and lower $\mathrm{FeO}$ and $\mathrm{TiO}_{2}$ contents with respect to MORB. The use of LFSE and $\mathrm{H}_{2} \mathrm{O}$ contents to test for a backarc basin origin for Site 828 basaltic rocks is precluded due to their strong alteration. However, $\mathrm{Nb}, \mathrm{P}, \mathrm{Zr}, \mathrm{Ti}$, and $\mathrm{Y}$ contents are lower than expected for MORB (Fig. 5), and are intermediate between the MORB and IAT averages of Sun (1980). As a result, Site 828 BABB have higher $\mathrm{Ti} / \mathrm{Zr}$ (around 200; Fig. 4) and lower $\mathrm{Zr} / \mathrm{Y}$ (0.92-2.3) than N-MORB, and $\mathrm{Y} / \mathrm{Nb}(13-20)$ tends to be higher than the N-MORB average (9-15; Cameron, 1985). $\mathrm{Pb}$ isotopic ratios also indicate a more radiogenic source than that of normal-MORB (Fig. 7). Hence, major elements, HFSE abundances, and isotopic data of these volcanics indicate a MORB/IAT transitional magmatic affinity, analogous to that of BABB. Group A2 Pic-BABB are not suitable parental magmas for Group A1 BABB, as they have higher Zr (almost double), $\mathrm{Ba}$, and $\mathrm{Sr}$ contents at comparable Ti contents.

The origin of these intermediate MORB-IAT compositional features of BABB is variably attributed to (1) mixing between normalMORB and/or enriched-MORB with IAT magmas (Ikeda and Yuasu, 1989); (2) introduction of an alkali-enriched component into the mantle below the back-arc (Price et al., 1990); or (3) addition of fluid/melt from the subducted slab (Sinton and Fryer, 1987). Whatever their petrogenetic scenario, the overall petrological features of these Site 828 volcanics support the hypothesis that their mantle source was significantly modified by subduction-related components, the influence of which was not strong enough to produce a typical IAT magmatism. Based on incompatible element distributions and their ratios, such as $\mathrm{Ti} / \mathrm{Zr}, \mathrm{Zr} / \mathrm{Nb}$, and $\mathrm{Zr} / \mathrm{Y}$, Group A BABB and Group B MORB magmas are not possibly comagmatic, or from the same mantle source. The BABB source was likely to be more refractory, and more enriched by fluid-transported LILE, than the MORB source.

$\mathrm{K} / \mathrm{Ar}$ age determinations on Sample 134-828A-15N-1, 120-122 $\mathrm{cm}$, and Sample 134-828A-15N-2, $0-4 \mathrm{~cm}$, yield ages of $18.7 \pm 2 \mathrm{Ma}$ and $10.6 \pm 1 \mathrm{Ma}$, respectively (Rex, this volume). However, a stratigraphic hiatus during most of the Miocene is present in the area and the few upper Oligocene (Rex, this volume; Baker et al., this volume) and middle Miocene (Collot et al., 1992) K/Ar ages recorded have so far been disregarded and attributed to Ar loss during alteration. In fact, the few planktonic foraminifers found in the volcanic breccia of Unit IV indicate a middle Eocene age. This age is also in agreement with the $56 \mathrm{Ma}$ (Paleocene-Eocene) and $36 \mathrm{Ma}$ (Eocene-Oligocene) fission track ages determined by Maillet et al. (1983) on volcanics dredged on the western part of DEZ. Volcanic rocks recovered at Bougainville Guyot (Site 831; Baker et al., this volume) have been dated from early Oligocene (36 $\pm 1 \mathrm{Ma}$; Sample 134-831B-84R-1, 50-58 cm) to Upper Oligocene (26.6 $\pm 1 \mathrm{Ma}$, Sample 134-831B-73R-2, 30-36 cm). Paleontological evidence based on foraminifers found in the overlying carbonate rocks has suggested a late Oligocene age (23.6-28.2 Ma; Collot, Green, Stokking, et al., 1992), but a ${ }^{87} \mathrm{Sr} /{ }^{86} \mathrm{Sr}$ age determination on the same carbonate yielded an age of $36.5 \mathrm{Ma}$ (Quinn et al., this volume). An age of $15 \mathrm{Ma}$ was also determined on a breccia dredged at Bougainville Guyot (Collot et al., 1992), but was considered unreliable due to the high alteration state of the rocks.

The two K/Ar determinations on the Bougainville basement rocks were carried out on samples only about $80 \mathrm{~m}$ apart, without any significant evidence of interruption in volcanic activity. Hence, Baker et al. (this volume) disregarded the $27 \mathrm{Ma}$ age on the ground that it probably resulted from Ar loss during alteration, and considered the $37 \pm 1 \mathrm{Ma}$ as reflecting the most likely age of the drilled volcanic sequence. Previous drilling by DSDP at Site 286 in the northern part of the Loyalty Basin recovered volcanic conglomerate (Stoeser, 1975) considered to have derived from the Bougainville Guyot (Fisher, 1986; Collot et al., 1992), and dated as between middle and late Eocene in age. 
Taking all the above factors into account, a Miocene age for Site 828 basaltic rocks appears unlikely, and, as for Site 831 lavas, the anomalously young ages are probably due to Ar loss during alteration of the Site 828 basalts. Hence, volcanism along the NDZ was probably active between 56 (Maillet et al., 1983) and $37 \mathrm{Ma}$ (Baker et al., this volume).

The tectono-magmatic setting of the NDR volcanics is another controversial matter. During the early Eocene to early Oligocene, a southwest-facing island arc existed in this region, as testified by arc lavas intercalated with middle Eocene sediments along the west coast of New Caledonia (Kroenke, 1984). If the DEZ represents the northern extension of the Norfolk Ridge, northeastward Cainozoic subduction was presumably active at that time beneath the NDR (Maillet et al., 1983; Kroenke, 1984). The relative motion of the Australia-India and Pacific plates suggests that along the DEZ this subduction was probably accompanied by large horizontal displacements, perhaps similar to that observed today at the Hunter Fracture Zone, along the southern termination of the New Hebrides Island Arc (Fig. 1). Andesitic rocks with island arc magmatic affinity from Bougainville Guyot and volcaniclastic rocks from Site 286 in the North Loyalty Basin may represent the products of this subduction episode. However, in this context, the MORB and BABB basalts recovered from the northern flank of the NDR are puzzling in that, if the SDC represented the volcanic arc of a northeastern-facing subduction zone, then Site 828 would be located on the forearc region of this arc. Recent marine geological and geophysical cruises and ODP drilling along the BoninMariana forearc and Lau-Tonga forearc have demonstrated that backarc opening may transect the arc, commencing in some parts of the arc within the arc axial chain, but sweeping into the forearc region or in other parts of the arc (e.g., Taylor, 1992). Magmas produced in the forearc region may be either generated from shallower, more refractory upper mantle peridotite, or segregate at deeper levels than in true backarc regions, thus traversing a longer melting column and consequently representing higher-degree partial melts. Either of these models might explain the unusually high-MgO, HFSE-depleted Group A2 magmas recorded from Site 828.

\section{CONCLUSIONS}

Lava breccias from below a middle Eocene foraminiferal ooze from Site 828 on the North d'Entrecasteaux Ridge include two basaltic magma suites. One suite, composed of aphyric to sparsely plagioclase + clinopyroxene-phyric basalts, is compositionally analogous to rather evolved normal MORB. The second group includes both basaltic and picritic (up to $15 \% \mathrm{MgO}$ ) lavas that have HFSE depletion and LILE enrichment relative to N-MORB, and major and trace element compositional features intermediate between N-MORB and IAT. These are compositionally akin to many backarc basin basalts (BABB). However considerations of the regional tectonic setting of the d'Entrecasteaux Ridge during the Eocene suggest that the spreading that generated these BABB may have occurred in the forearc region of the southwest-facing arc, part of which probably is represented by Bougainville Guyot.

\section{ACKNOWLEDGMENTS}

Time aboard JOIDES Resolution for Coltorti was financially supported by C.N.R. (Italy). The authors thank B. Galassi, E. Condliffe, A. Fujinawa, and C. LaPorte for support in the analytical work. Improvements of the manuscript resulted from discussions with $\mathrm{L}$. Beccaluva; constructive reviews by Tony Crawford, Steve Eggins, and Jean-Philippe Eissen are gratefully appreciated. We also thank Tony Crawford for the invaluable overviews of the complex geological evolution of the New Hebrides Island Arc he gave us during the Leg 134 post-cruise meeting in Villefranche-sur-Mer (France).

\section{REFERENCES}

Alt, J.C., Honnorez, J., Laverne, C., and Emmermann, R., 1986. Hydrothermal alteration of a $1 \mathrm{~km}$ section through the upper oceanic crust, Deep Sea Drilling Project Hole 504B: mineralogy, chemistry, and evolution of seawater-basalt interactions. J. Geophys. Res., 91:10309-10335.

Beccaluva, L., Macciotta, G., Piccardo, G.B., and Zeda, O., 1989. Clinopyroxene composition of ophiolite basalts as petrogenetic indicators. Chem. Geol., 77:165-182.

Bienvenu, P., Bougault, H., Joron, J.L., Treuil, M., and Dmitriev, L., 1990. MORB alteration: rare-earth element/non-rare-earth hygromagmaphile element fractionation. Chem. Geol., 82:1-14.

Brown, R.W., Allsopp, H.L., Bristow, J.W., and Smith, B.G., 1989. Improved precision of $\mathrm{Rb}$-Sr dating of kimberlitic micas: an assessment of a leaching technique. Chem. Geol., 79:125-136.

Burne, R.V., Collot, J.-Y., and Daniel, J., 1988. Superficial structures and stress regimes of the downgoing plate associated with subduction-collision in the Central New Hebrides Arc (Vanuatu). In Greene, H.G., and Wong, F.L. (Eds.), Geology and Offshore Resources of Pacific Island Arcs-Vanuatu Region. Circum-Pac. Council Energy Miner. Resour., Earth Sci. Ser., 8:357-376.

Cameron, W.E., 1985. Petrology and origin of primitive lavas from the Troodos ophiolite, Cyprus. Contrib. Mineral. Petrol., 89:239-255.

Collot, J.-Y., Daniel, J., and Burne, R.V., 1985. Recent tectonics associated with the subduction/collision of the d'Entrecasteaux zone in the central New Hebrides. Tectonophysics, 112:325-356.

Collot, J.-Y., Greene, H.G., Stokking, L.B., et al., 1992. Proc. ODP, Init. Repts., 134: College Station, TX (Ocean Drilling Program).

Collot, J.-Y, Lallemand, S., Pelletier, B., Eissen, J.-P., Glaçon, G., Fisher, M.A., Greene, H.G., Boulin, J., Daniel, J., and Monzier, M., 1992. Geology of the d'Entrecasteaux-New Hebrides island arc collision: results from a deep-sea submersible survey. Tectonophysics, 212:213-241.

Collot, J.-Y., Pelletier, B., Boulin, J., Daniel, J., Eissen, J.P., Fisher, M.A., Greene, H.G., Lallemand, S., and Monzier, M., 1989. Premiers résultats des plongees de la campagnee SUBPSO1 dans la zone de collision des rides d'Entrecasteaux et de l'arc des Nouvelles Hébrides. C. R. Acad. Sci. Ser. 2, 309:1947-1954.

Dick, H.J.B., and Bullen, T., 1984. Chromian spinel as a petrogenetic indicator in abyssal and alpine-type peridotites and spatially associated lavas. Contrib. Mineral. Petrol., 86:54-76.

Elthon, D., 1981. Metamorphism in oceanic spreading centers. In Emiliani, C. (Ed.), The Sea (Vol. 7): The Oceanic Lithosphere: New York (Wiley), 285-303.

Falloon, T.J., Malahoff, A., Zonenshain, L.P., and Bogdanov, Y., 1992. Petrology and geochemistry of back-arc basin basalts from Lau Basin spreading ridges at 15,18 , and $19^{\circ} \mathrm{S}$. Mineral. Petrol., 47:1-35.

Fisher, M.A., 1986. Tectonic processes at the collision of the d'Entrecasteaux zone and the New Hebrides island arc. J. Geophys. Res., 91:10470-10486.

Franzini, M., Leoni, L., and Saitta, M., 1975. Revisione di una metodologia analitica per fluorescenza-X, basata sulla correzione completa degli effetti di matrice. Rend. Soc. Ital. Mineral. Petrol., 31:365-378.

Hart, S.R., Erlank, A.J., and Kable, J.D., 1974. Sea floor basalt alteration: some chemical and $\mathrm{Sr}$ isotopic effects. Contrib. Mineral. Petrol., 44:219-230.

Hawkins, J.W., and Melchior, J.T., 1985. Petrology of Mariana Trough and Lau Basin basalts. J. Geophys. Res., 90:11431-11468.

Ikeda, Y., and Yuasu, M., 1989. Volcanism in nascent back-arc basins behind the Shichito Ridge and adjacent areas in the Izu-Ogasawara arc, Northwest Pacific: evidence for mixing between E-type MORB and island arc magmas at the initiation of backarc rifting. Contrib. Mineral. Petrol., 101:377-393.

Kroenke, L.W., 1984. Cenozoic Tectonic Development of the Southwest Pacific. Tech. Bull.-U.N. Econ. Soc. Comm. Asia Pac., Comm. Co-ord. Jt. Prospect Miner. Resour. South Pac. Offshore Areas, 6.

Leoni, L., and Saitta, M., 1976. X-ray flourescence analyses of 29 trace elements in rock and mineral standards. Rend. Soc. Ital. Mineral. Petrol., $32: 497-510$

\footnotetext{
Abbreviations for names of organizations and publications in ODP reference lists follow the style given in Chemical Abstracts Service Source Index (published by American Chemical Society).
} 
Maillet, P., Monzier, M., Selo, M., and Storzer, D., 1983. The d'Entrecasteaux zone (southwest Pacific). A petrological and geochronological reappraisal. Mar. Geol., 53:179-197.

Menzies, M.A., and Seyfried, W.E., 1979. Basalt-seawater interaction: trace element and strontium isotopic variation in experimentally altered glassy basalt. Earth Planet. Sci. Lett., 44:463-472.

Mottl, M.J., and Holland, H.D., 1978. Chemical exchange during hydrothermal alteration of basalt by seawater. I. Experimental results for major and minor components of seawater. Geochim. Cosmochim. Acta, 42:1103-1115.

Philpotts, J.A., Schnetzler, C.C., and Hart, S.R., 1969. Submarine basalts: some $\mathrm{K}, \mathrm{Rb}, \mathrm{Sr}, \mathrm{Ba}$, rare-earth, $\mathrm{H}_{2} \mathrm{O}$, and $\mathrm{CO}_{2}$ data bearing on their alteration, modification by plagioclase, and possible source materials. Earth Planet. Sci. Lett., 7:293-299.

Price, R.C., Johnson, L.E., and Crawford, A.J., 1990. Basalts of the North Fiji Basin: the generation of back arc basin magmas by mixing of depleted and enriched mantle sources. Contrib. Mineral. Petrol., 105:106-121.

Reynolds, R.C., 1967. Estimations of mass absorption coefficients by Compton scattering: improvements and extensions of the method. Am. Mineral., 48:1133-1143.

Roelandts, I., and Michel, G., 1986. Sequential inductively coupled plasma determination of some rare-earth elements in five french geostandards. Geostand. Newsl., 10:135-154.

Sinton, J.M., and Fryer, P., 1987. Mariana Trough lavas from $18^{\circ} \mathrm{N}$ : implications for the origin of back arc basin basalts. J. Geophys. Res., 92:1278212802.

Spadea, P., Beccaluva, L., Civetta, L., Coltorti, M., Dostal, J., Sajona, F., Serri, G., Vaccaro, C., and Zeda, O., 1991. Petrology of basic igneous rocks from the floor of the Sulu Sea. In Silver, E.A., Rangin, C., von Breymann, M.T. et al., Proc. ODP, Sci. Results, 124: College Station, TX (Ocean Drilling Program), 251-270.

Stoeser, D.B., 1975. Igneous rocks from Leg 30 of the Deep Sea Drilling Project. In Andrews, J.E., Packham, G., et al., Init. Repts. DSDP, 30: Washington (U.S. Govt. Printing Office), 401-414.

Sun, S.-S., 1980. Lead isotopic study of young volcanic rocks from mid-ocean ridges, ocean islands and island arcs. Philos. Trans. R. Soc. London A 297:409-445.
Sun, S.-S., and McDonough, W.F., 1989. Chemical and isotopic systematics of oceanic basalts: implications for mantle composition and processes. In Saunders, A.D., and Norry, M.J. (Eds.), Magmatism in the Ocean Basins. Geol. Soc. Spec. Publ. London, 42:313-345.

Sun, S.-S., Nesbitt, R.W., and Sharaskin, A.Y., 1979. Geochemical characteristics of mid-ocean ridge basalts. Earth Planet. Sci. Lett., 44:119-138.

Taylor, B., 1992. Rifting and the volcanic-tectonic evolution of the Izu-BoninMariana Arc. In Taylor, B., Fujioka, K., et al., Proc. ODP, Sci. Results, 126: College Station, TX (Ocean Drilling Program), 627-651.

Viereck, L.G., Flower, M.F.J., Hertogen, J., Schmincke, H.-U., and Jenner, G.A., 1989. The genesis and significance of N-MORB sub-types. Contrib. Mineral. Petrol., 102:112-126.

Volpe, A.M., Macdougall, J.D., and Hawkins, J.W., 1988. Lau basin basalts (LBB): trace element and $\mathrm{Sr}$-Nd isotopic evidence for heterogeneity in backarc basin mantle. Earth Planet. Sci. Lett., 90:174-186.

Volpe, A.M., Macdougall, J.D., and Lugmair, G.W., 1988. Pb isotopes in back-arc basin basalts from the Mariana Trough and Lau Basin. Eos, 69:1471.

White, W.M., and Dupré, B., 1986. Sediment subduction and magma genesis in the Lesser Antilles: isotopic and trace element constraints. J. Geophys. Res., 91:5927-5941.

Wilson, M., 1989. Igneous Petrogenesis: A Global Tectonic Approach: London (Unwin Hyman).

Wood, D.A., Tarney, J., Varet, J., Saunders, A.D., Bougault, H., Joron, J.-L., Treuil, M., and Cann, J.R., 1979. Geochemistry of basalts drilled in the North Atlantic by IPOD Leg 49: implications for mantle heterogeneity. Earth Planet. Sci. Lett., 42:77-97.

Date of initial receipt: 24 April 1992

Date of acceptance: 17 June 1993

Ms 134SR-016 